\title{
Self-similar sets: Projections, Sections and Percolation
}

\author{
Kenneth Falconer and Xiong Jin
}

\begin{abstract}
We survey some recent results on the dimension of orthogonal projections of self-similar sets and of random subsets obtained by percolation on self-similar sets. In particular we highlight conditions when the dimension of the projections takes the generic value for all, or very nearly all, projections. We then describe a method for deriving dimensional properties of sections of deterministic self-similar sets by utilising projection properties of random percolation subsets.
\end{abstract}

\section{Introduction}

Throughout this article we will work with subsets of the Euclidean plane $\mathbb{R}^{2}$, though some, but not all, of the results presented have higher dimensional analogues. Relating the Hausdorff dimension $\operatorname{dim}_{H} E$ of a set $E \subset \mathbb{R}^{2}$ to the dimensions of its projections and sections has a long history. Let $\operatorname{proj}_{\theta}$ denote orthogonal projection onto the line $L_{\theta}$ in direction $\theta$, and write $\mathscr{L}$ for Lebsegue measure on $L_{\theta}$ (see Fig. 1). Marstrand's projection theorem is well-known.

Theorem 1.1. [25] Let $E \subset \mathbb{R}^{2}$ be a Borel set.

(i) For all $\theta \in[0, \pi)$

$\operatorname{dim}_{H} \operatorname{proj}_{\theta} E \leq \min \left\{\operatorname{dim}_{H} E, 1\right\}$.

(ii) For Lebsegue almost all $\theta \in[0, \pi)$

$\operatorname{dim}_{H} \operatorname{proj}_{\theta} E=\min \left\{\operatorname{dim}_{H} E, 1\right\}$.

(iii) If $\operatorname{dim}_{H} E>1$ then for Lebesgue almost all $\theta \in[0, \pi)$

$$
\mathscr{L}\left(\operatorname{proj}_{\theta} E\right)>0 \text {. }
$$

Kenneth Falconer

Mathematical Institute, University of St Andrews, North Haugh, St Andrews, Fife, KY16 9SS,

United Kingdom, e-mail: kjf@st-andrews.ac.uk

Xiong Jin

School of Mathematics, Alan Turing Building, The University of Manchester, Manchester, M13

9PL, United Kingdom e-mail: xiong.jin@manchester.ac.uk 

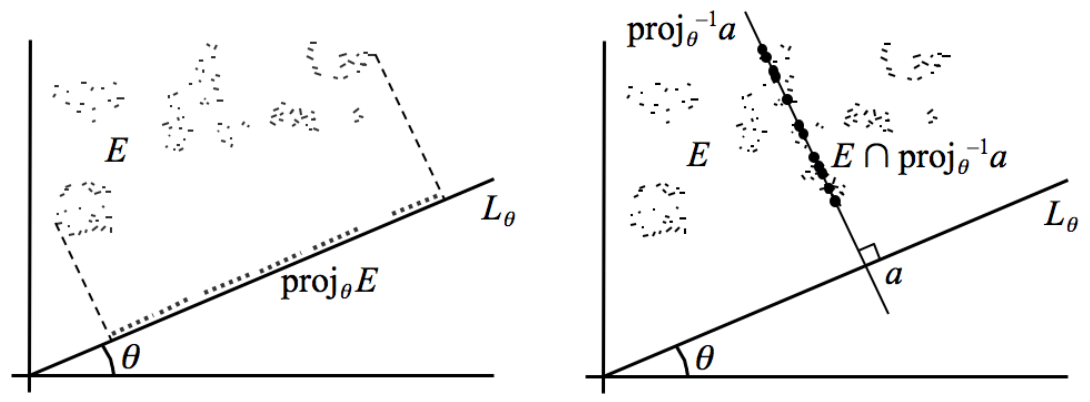

Fig. 1 Projection $\operatorname{proj}_{\theta} E$ and section $E \cap \operatorname{proj}_{\theta}^{-1} a$ of a set $E$

There are related results for sections of sets, where $E \cap \operatorname{proj}_{\theta}^{-1} a$ is the section or slice of $E$ by the line perpendicular to $L_{\theta}$ that passes through $a \in L_{\theta}$.

Theorem 1.2. $[25,26]$ Let $E \subset \mathbb{R}^{2}$ be a Borel set with $\operatorname{dim}_{H} E>1$.

(i) For all $0 \leq \Delta \leq 1$ and all $\theta \in[0, \pi)$

$$
\Delta+\operatorname{dim}_{H}\left\{a \in L_{\theta}: \operatorname{dim}_{H}\left(E \cap \operatorname{proj}_{\theta}^{-1} a\right) \geq \Delta\right\} \leq \operatorname{dim}_{H} E .
$$

(ii) In particular, for all $\theta \in[0, \pi)$

$\operatorname{dim}_{H}\left(E \cap \operatorname{proj}_{\theta}^{-1} a\right) \leq \operatorname{dim}_{H} E-1$ for $\mathscr{L}$-almost all $a \in L_{\theta}$.

(iii) If $\operatorname{dim}_{H} E>1$ then for all $\varepsilon>0$ and Lebesgue almost all $\theta \in[0, \pi)$, $\mathscr{L}\left\{a \in L_{\theta}: \operatorname{dim}_{H}\left(E \cap \operatorname{proj}_{\theta}^{-1} a\right) \geq \operatorname{dim}_{H} E-1-\varepsilon\right\}>0$.

(Note that we take $\operatorname{dim}_{H} \emptyset=-\infty$ ).

These inequalities were obtained by Marstrand and extended to subsets of $\mathbb{R}^{d}$ for $d>2$ by Mattila [27]. Kaufman [20] introduced the potential theoretic method now generally used in studying dimensions of projections and sections of sets, see [28] for a good exposition and proofs of these results.

In general, Marstrand's projection Theorem 1.1 tells nothing about which particular directions $\theta$ may have projections with dimension or measure smaller than normal. However, the set of exceptional directions cannot be too big.

Theorem 1.3. [5, 20] Let $E \subset \mathbb{R}^{2}$ be a Borel set.

(i) If $\operatorname{dim}_{H} E \leq 1$ then

$$
\operatorname{dim}_{H}\left\{\theta: \operatorname{dim}_{H} \operatorname{proj}_{\theta} E<\operatorname{dim}_{H} E\right\} \leq \operatorname{dim}_{H} E .
$$

(ii) If $\operatorname{dim}_{H} E>1$ then

$$
\operatorname{dim}_{H}\left\{\theta: \mathscr{L}\left(\operatorname{proj}_{\theta} E\right)=0\right\} \leq 2-\operatorname{dim}_{H} E .
$$

Part (i) was obtained by Kaufman [20] using an energy estimate and part (ii) by Falconer [5] using Fourier transforms. Nevertheless, identifying the exceptional $\theta$, if any, remains problematic even for familiar fractal sets.

More recently, Fursternberg [10] introduced the notion of dimension conservation for when an opposite inequality to Theorem 1.2(i) holds. A projection $\operatorname{proj}_{\theta}$ is said to be dimension conserving for $E \subset \mathbb{R}^{2}$ if there is a number $\Delta>0$ such that 


$$
\Delta+\operatorname{dim}_{H}\left\{a \in L_{\theta}: \operatorname{dim}_{H}\left(E \cap \operatorname{proj}_{\theta}^{-1} a\right) \geq \Delta\right\} \geq \operatorname{dim}_{H} E .
$$

Fursternberg [10] showed that various subsets of the plane, including certain selfsimilar sets, are dimension conserving for all $\theta$, that is there are no exceptional directions for (1).

We will consider a slightly weaker property in the case $\operatorname{dim}_{H} E>1$. We call a projection $\operatorname{proj}_{\theta}$ weakly dimension conserving if, for all $\varepsilon>0$,

$$
\operatorname{dim}_{H}\left(E \cap \operatorname{proj}_{\theta}^{-1} a\right) \geq \operatorname{dim}_{H} E-1-\varepsilon \quad \text { for all } a \in A,
$$

where $A$ is a 'large' subset of $L_{\theta}$, either with $\operatorname{dim}_{H} A=1$ or with $\mathscr{L}(A)>0$. It follows from Theorem 1.2(iii) that $\operatorname{proj}_{\theta}$ is weakly dimension conserving for Lebesgue almost every $\theta \in[0, \pi)$.

There has been great interest recently in identifying classes of sets, especially self-similar sets and their variants, for which the conclusions of these theorems hold for all $\theta$ rather than just almost all $\theta$. For example, the conclusions of Theorem 1.1 hold for all projections for certain classes of self-similar sets [10, 15, 31, 36] as well as for random subsets of certain self-similar sets [7, 33, 35, 34, 38].

Some of these results will be surveyed in this article. However, our main aim is to indicate a method that enables dimension results for sections of deterministic sets to be derived from projection properties of random percolation sets. In particular, many self-similar sets are weakly dimension conserving for all or very nearly all $\theta$.

\section{Projections of self-similar sets}

Let $f_{1}, \ldots, f_{m}: \mathbb{R}^{2} \rightarrow \mathbb{R}^{2}$ be an iterated function system (IFS) of contracting similarities. By standard IFS theory of IFS there exists a unique non-empty compact $E \subset \mathbb{R}^{2}$ such that

$$
E=\bigcup_{i=1}^{m} f_{i}(E)
$$

the set $E$ is termed a self-similar set, see $[6,16]$. We may write the similarities as

$$
f_{i}(x)=r_{i} O_{i}(x)+t_{i}
$$

where $0<r_{i}<1$ is the scale factor, $O_{i}$ is a rotation (we assume for convenience that the $f_{i}$ are orientation preserving) and $t_{i}$ is a translation. The IFS $\left\{f_{1}, \ldots, f_{m}\right\}$ is said to have dense rotations if at least one of the $O_{i}$ is a rotation by an irrational multiple of $\pi$, equivalently if group $\left\{O_{1}, \ldots, O_{m}\right\}$ is dense in $S O(2, \mathbb{R})$. Otherwise $\left\{f_{1}, \ldots, f_{m}\right\}$ has finite rotations (see Fig. 2).

Provided the strong separation condition (SSC) (i.e. the union in (3) is disjoint) or the open set condition (OSC) (i.e. there is a non-empty open set $O$ such that $O \supset$ $\bigcup_{i=1}^{m} f_{i}(O)$ with the union disjoint) holds, then $\operatorname{dim}_{H} E=s$, where $s$ is the similarity dimension given by $\sum_{i=1}^{m} r_{i}^{s}=1$. 


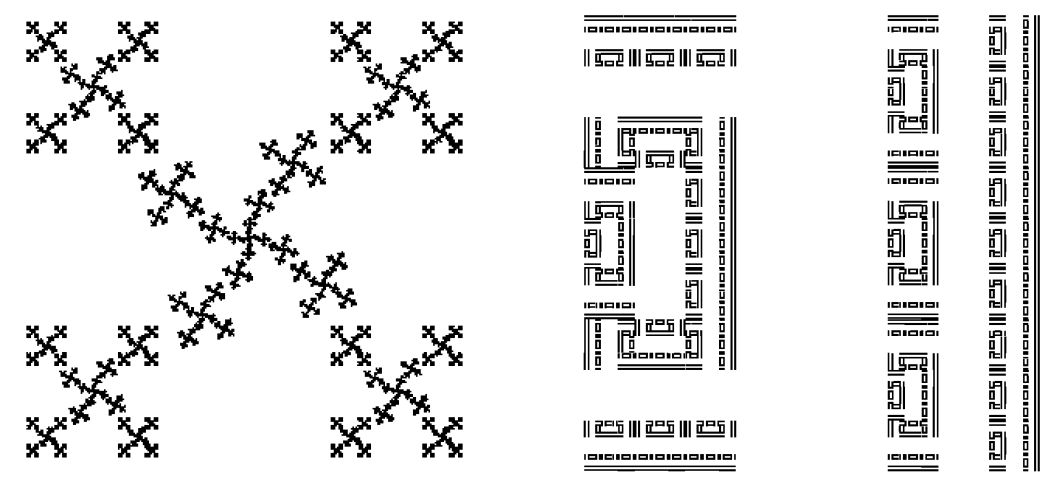

Fig. 2 A self-similar set with dense rotations and one with finite rotations

We may easily construct self-similar sets with a finite rotation group $G$ for which the conclusions of Marstrand's theorem fail in certain directions. For example, let $f_{1}, \ldots, f_{4}$ be homotheties (i.e. similarities with $O_{i}$ the identity in (4)) of ratio $0<r<\frac{1}{4}$ that map the unit square $S$ into itself, each $f_{i}$ fixing one of the four corners. Then $\operatorname{dim}_{H} E=-\log 4 / \log r$, but the projections of $E$ onto the sides of the square have dimension $-\log 2 / \log r$ and onto the diagonals of $S$ have dimension $-\log 3 / \log r$, a consequence of the alignment of the component squares $f_{i}(S)$ under projection. Indeed, for any IFS consisting of homotheties there is a similar reduction in the dimension of projections in direction $\theta$ whenever $\operatorname{proj}_{\theta}\left(f_{i_{1}} \circ \cdots \circ f_{i_{k}}(S)\right)=$ $\operatorname{proj}_{\theta}\left(f_{j_{1}} \circ \cdots \circ f_{j_{k}}(S)\right)$ for some distinct words $i_{1}, \ldots, i_{k}$ and $j_{1}, \ldots, j_{k}$.

Kenyon [21] investigated the projections of the 1-dimensional Sierpiński gasket $E \subset \mathbb{R}^{2}$, that is the self-similar set defined by the similarities

$$
f_{1}(x, y)=\left(\frac{1}{3} x, \frac{1}{3} y\right), f_{2}(x, y)=\left(\frac{1}{3} x+\frac{2}{3}, \frac{1}{3} y\right), f_{3}(x, y)=\left(\frac{1}{3} x, \frac{1}{3} y+\frac{2}{3}\right) .
$$

He showed that the projection of $E$ onto a line of slope $p / q$ with has dimension strictly less than 1 if $p+q \neq \equiv 0(\bmod 3)$, but if $p+q \equiv 0(\bmod 3)$ then the projection has non-empty interior. He also proved that the projections onto lines with irrational slope have Lebesgue measure 0 and Hochman [14] further showed that they nevertheless have Hausdorff dimension 1.

Sections and projections of other specific self-similar sets have been analysed in $[2,22,24]$.

In fact, when the rotation group is finite, there are always some projections for which direct overlapping of the projection of components of the construction leads to a dimension drop, as the following theorem of Farkas shows.

Theorem 2.1. [9] If $E \subset \mathbb{R}^{2}$ is self-similar with finite rotation group and similarity dimension $s$, then $\operatorname{dim}_{H} \operatorname{proj}_{\theta} E<s$ for at least one $\theta \in[0, \pi)$. In particular if $E$ satisfies OSC and $0<\operatorname{dim}_{H} E<1$ then $\operatorname{dim}_{H} \operatorname{proj}_{\theta} E<\operatorname{dim}_{H} E$ for some $\theta$.

A rather different situation occurs if the IFS has dense rotations. 
Theorem 2.2. $[15,31]$ If $E \subset \mathbb{R}^{2}$ is self-similar with dense rotations then

$$
\operatorname{dim}_{H} \operatorname{proj}_{\theta} E=\min \left\{\operatorname{dim}_{H} E, 1\right\} \text { for all } \theta \in[0, \pi) .
$$

Peres and Shmerkin [31] proved this in the plane without requiring any separation condition on the IFS. For this they set up a discrete version of Marstrand's projection theorem to construct a tree of intervals in the line $L_{\theta}$ and then apply Weyl's equidistribution theorem. Hochman and Shmerkin [15] gave a very different proof, also valid in higher dimensions. Their proof uses the CP-chains of Furstenberg [10,12], see also [13], and has three main ingredients: the lower semicontinuity of the expected Hausdorff dimension of the projection of a measure with respect to its 'micromeasures', Marstrand's projection theorem, and the invariance of the dimension of projections under the action of the rotation group.

The open set condition is not essential here since, for all $\varepsilon>0$, a Vitali argument may be used to set up a new IFS, consisting of certain compositions of the $f_{i}$, that satisfies SSC, with attractor $E^{\prime} \subset E$ such that $\operatorname{dim}_{H} E^{\prime}>\operatorname{dim}_{H} E-\varepsilon$; we can also ensure that the new IFS has dense rotations if the original one has, see [7, 9, 29, 31].

In the dense rotation case, if $\operatorname{dim}_{H} E>1$ then $\operatorname{dim}_{H} \operatorname{proj}_{\theta} E=1$ for all $\theta$ by Theorem 2.2, but in view of Theorem 1.1(iii) we might hope that the projections also have positive Lebesgue measure. Shmerkin and Solomyak showed that this is very nearly so.

Theorem 2.3. [36] Let $E \subset \mathbb{R}^{2}$ be the self-similar attractor of an IFS with dense rotations with $1<\operatorname{dim}_{H} E<2$. Then $\mathscr{L}\left(\operatorname{proj}_{\theta} E\right)>0$ for all $\theta$ except (perhaps) for a set of $\theta$ of Hausdorff dimension 0.

The proof depends on careful estimation of the decay of the Fourier transforms of projections of a measure supported by $E$ and how this varies with direction. Components of the method can be traced back to a study of Bernoulli convolutions by Erdós [4], which Kahane [17] pointed out gave an exceptional set of Hausdorff dimension 0 rather than just Lebesgue measure 0 , see [30].

\section{Projections of percolation sets}

Fractal percolation is a process that leads to statistically self-similar subsets of a given self-similar set, with the same random mechanism determining the form of the fractals at both large and small scales.

Best known is Mandelbrot percolation, involving repeated splitting of squares into subsquares from which sub-collections are randomly selected. Let $E \subset \mathbb{R}^{2}$ be the unit square. Fix an integer $M \geq 2$ and a probability $0<p<1$. We divide $E$ into $M^{2}$ closed subsquares of side $1 / M$ in the obvious way, and retain each subsquare independently with probability $p$ to get a set $E^{1}$ formed as a union of the retained subsquares. We repeat this process on the squares of $E^{1}$, dividing each into $M^{2}$ subsquares of side $1 / M^{2}$ and retaining each with probability $p$ to get a set $E^{2}$, and 

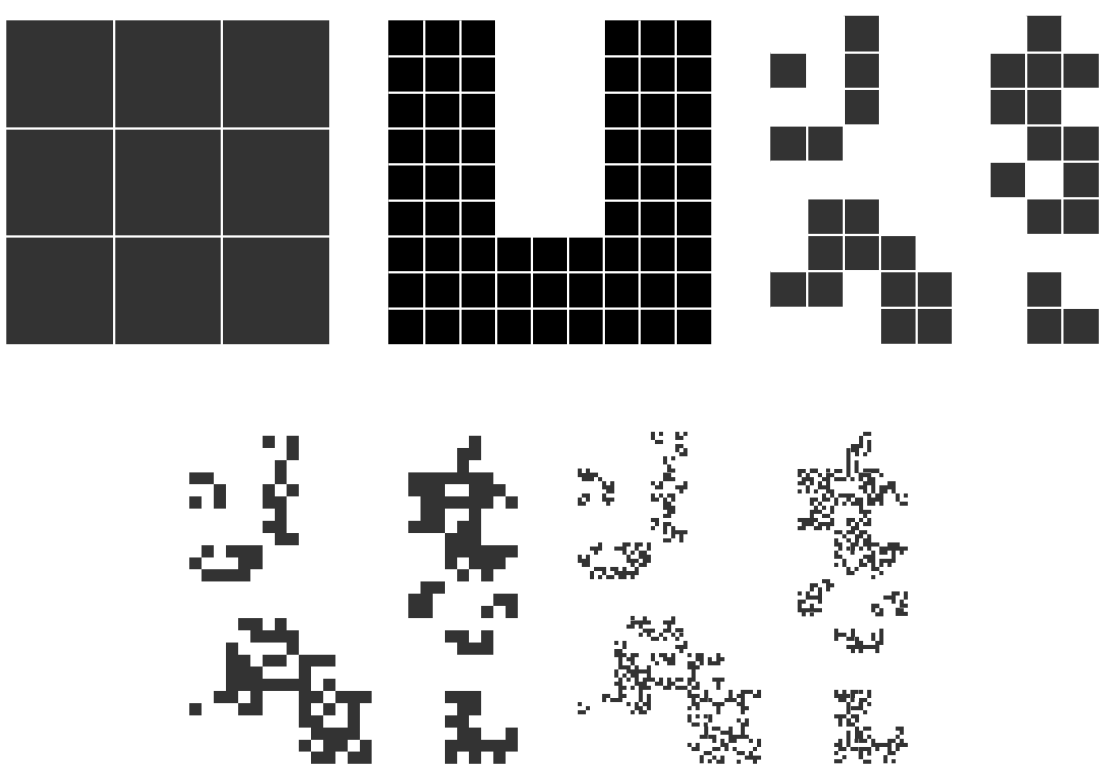

Fig. 3 Mandelbrot percolation on the unit square with $3 \times 3$ subdivision

we continue in this way (see Fig. 3). This leads to the random percolation set $E_{p}=$ $\bigcap_{k=0}^{\infty} E^{k}$. If $p>1 / M^{2}$ then by a branching process argument there is a positive probability of non-extinction, i.e. that $E_{p} \neq \emptyset$, conditional on which $\operatorname{dim}_{H} E_{p}=2+$ $\log p / \log M$ almost surely.

The topological properties of Mandelbrot percolation have been extensively studied, see $[3,6,34]$. In particular, there is a critical probability $p_{c}$ with $1 / M<p_{c}<1$ such that if $p>p_{c}$ then, conditional on non-extinction, $E_{p}$ contains many connected components, so its projections onto all lines necessarily have positive Lebesgue measure. If $p \leq p_{c}$ the percolation set $E_{p}$ is totally disconnected, and Marstrand's theorems provide information on its projections in almost all directions. However, Rams and Simon $[33,34,35]$ recently showed using a careful geometrical analysis that, conditional on $E_{p} \neq \emptyset$, almost surely the conclusions of Theorem 1.1 hold for all projections simultaneously.

Theorem 3.1. [33] Let $E_{p}$ be the random Mandelbrot percolation set obtained based on subdivision of squares into $M^{2}$ subsquares, each square being retained with probability $p>1 / M^{2}$. Then, with positive probability, $E_{p} \neq \emptyset$, conditional on which:

(i) $\operatorname{dim}_{H} \operatorname{proj}_{\theta} E_{p}=\min \left\{\operatorname{dim}_{H} E_{p}, 1\right\}$ for all $\theta \in[0, \pi)$,

(ii) if $p>1 / M$ then for all $\theta \in[0, \pi)$, $\operatorname{proj}_{\theta} E_{p}$ contains an interval and in particular $\mathscr{L}\left(\operatorname{proj}_{\theta} E_{p}\right)>0$. 


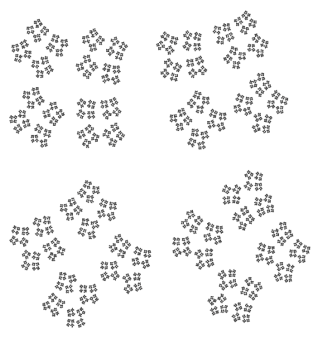

E

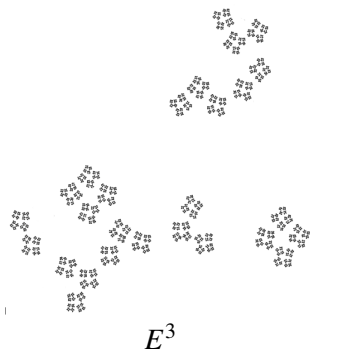

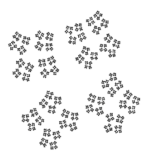

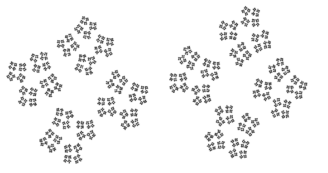

$E^{1}$
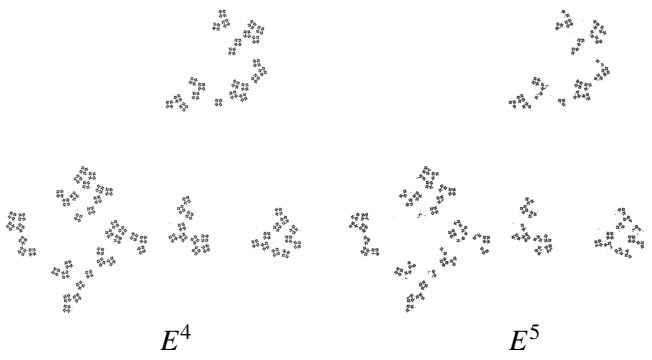

Fig. 4 Percolation on a self-similar fractal $E$

A similar percolation process may be carried out on any self-similar set. Let $\left\{f_{1}, \ldots, f_{m}\right\}$ be an IFS of similarities on $\mathbb{R}^{2}$ defining a self-similar set $E$ satisfying (3). Components of the natural hierarchical construction of $E$ are retained or deleted in a random but self-similar manner as follows. Let $\mathbf{p}=\left(p_{1}, \ldots, p_{m}\right)$ be a vector of probabilities where $0 \leq p_{i}<1(1 \leq i \leq m)$. We first select a random subfamily of the sets $\left\{f_{1}(E), \ldots, f_{m}(E)\right\}$ according to some distribution, with $f_{i}(E)$ selected with probability $p_{i}$ for each $i$, and write $E^{1}$ for the union of the selected sets. Then, for each selected $f_{i}(E)$, we select subsets $\left\{f_{i} f_{1}(E), \ldots, f_{i} f_{m}(E)\right\}$ with the same distribution as before and independently for each $i$; the union of these sets comprising $E^{2}$. Continuing in this way, we get a nested hierarchy $E \supset E^{1} \supset E^{2} \supset \cdots$ of random compact sets, where $E^{k}$ is the union of the components remaining at the $k$ th stage (see Fig. 4). We write $E_{\mathbf{p}}=\bigcap_{k=1}^{\infty} E^{k}$ for the random percolation subset of $E$ determined by the probabilities $\mathbf{p}$. Provided that $\sum_{i=1}^{m} p_{i}>1$ there is a positive probability that $E_{\mathbf{p}} \neq \emptyset$, conditional on which $\operatorname{dim}_{H} E_{\mathbf{p}}$ is given by the number $s>0$ satisfying $\sum_{i=1}^{m} r_{i}^{s} p_{i}=1$, see for example [6].

In the case when the IFS defining $E$ has dense rotations the ergodic theoretic methods of [15] may be extended to random cascade measures to provide a random analogue of Theorem 2.2.

Theorem 3.2. [7] Let $E \subset \mathbb{R}^{2}$ be a self-similar set with dense rotations satisfying the open set condition and let $E_{\mathbf{p}} \subset E$ be the percolation set described above. If $\sum_{i=1}^{m} p_{i}>1$ then $E_{\mathbf{p}} \neq \emptyset$ with positive probability, conditional on which, 


$$
\operatorname{dim}_{H} \operatorname{proj}_{\theta} E_{\mathbf{p}}=\min \left\{\operatorname{dim}_{H} E_{\mathbf{p}}, 1\right\} \text { for all } \theta \in[0, \pi) .
$$

Theorem 3.2 is a special case of a more general result on a random multiplicative cascade measure $\mu$ constructed on a self-similar set $E$ as follows.

Let

$$
W=\left(W_{1}, \ldots, W_{m}\right) \in[0, \infty)^{m}
$$

be a random vector such that $\sum_{i=1}^{m} \mathbb{E}\left(W_{i}\right)=1$. For $k \geq 0$ and $\left(i_{1}, \ldots, i_{k}\right) \in\{1, \ldots, m\}^{k}$ let

$$
W^{i_{1}, \ldots, i_{k}}=\left(W_{1}^{i_{1}, \ldots, i_{k}}, \ldots, W_{m}^{i_{1}, \ldots, i_{k}}\right) \in[0, \infty)^{m}
$$

be independent identically distributed copies of $W$. The condition $\sum_{i=1}^{m} \mathbb{E}\left(W_{i}\right)=1$ ensures that for each $i_{1}, \ldots, i_{j}$ the sequence $\left(\mu_{k}\left(f_{i_{1}} \circ \cdots \circ f_{i_{j}}(E)\right)\right)_{k \geq j}$ given by

$$
\begin{aligned}
& \mu_{k}\left(f_{i_{1}} \circ \cdots \circ f_{i_{j}}(E)\right) \\
& \quad \equiv W_{i_{1}} W_{i_{2}}^{i_{1}} W_{i_{3}}^{i_{1}, i_{2}} \cdots W_{i_{j}}^{i_{1}, \ldots, i_{j-1}} \sum_{1 \leq i_{j+1}, \ldots, i_{k} \leq m} W_{i_{j+1}}^{i_{1}, \ldots, i_{j}} \cdots W_{i_{k}}^{i_{1}, \ldots, i_{k-1}}
\end{aligned}
$$

is a martingale, so $\mu_{k}\left(f_{i_{1}} \circ \cdots \circ f_{i_{j}}(E)\right)$ converges almost surely to some number $\mu\left(f_{i_{1}} \circ \cdots \circ f_{i_{j}}(E)\right) \geq 0$. Since each $\mu_{k}$ is additive on the sets $\left\{f_{i_{1}} \circ \cdots \circ f_{i_{j}}(E): 1 \leq\right.$ $\left.i_{j} \leq m, j \leq k\right\}$, it follows that $\mu$ is additive on all finite unions of such sets and so extends to a measure $\mu$ on the Borel $\sigma$-algebra generated by these sets. Thus $\mu$ is a random measure supported by $E$ termed a random multiplicative cascade measure. Moreover, provided that $\sum_{i=1}^{m} \mathbb{E}\left(\left(W_{i}\right)^{p}\right)<\infty$ for some $p>1$, then $\mu$ is non-trivial, i.e. $\mu(E)>0$ with positive probability, see [1].

This construction includes several special cases:

- Mandelbrot multiplicative cascades (when the $W_{i}(1 \leq i \leq m)$ are independent identically distributed with the distribution of some $W_{0}$ where $\mathbb{E}\left(W_{0}\right)=1$, introduced by Mandelbrot [23] and studied in detail by Kahane, Peyrière and others $[18,19,32])$;

- Natural measures on fractal percolation sets (where each $W_{i}$ independently takes values of either 0 or some positive number);

- Branching constructions (where $W=\left(W_{1}, \ldots, W_{m}\right)$ is a random vector where each component $W_{i}$ is either 0 or some constant number).

The following theorem extends Theorem 2.2 to projections of random multiplicative cascades; recall that the projection of a measure $v$ on $\mathbb{R}^{2}$ onto the line $L_{\theta}$ is given by $\left(\operatorname{proj}_{\theta} v\right)(A)=v\left\{x \in \mathbb{R}^{2}: \operatorname{proj}_{\theta} x \in A\right\}$ for $A \subset L_{\theta}$. Note that we do not require any separation condition.

Theorem 3.3. [7] Let $\mu$ be a random multiplicative cascade measure on a selfsimilar set $E \subset \mathbb{R}^{2}$ with dense rotations. Then almost surely, conditional on $\mu \neq 0$,

$$
\operatorname{dim}_{H} \operatorname{proj}_{\theta} \mu=\min \left\{\operatorname{dim}_{H} \mu, 1\right\} \text { for all } \theta \text {. }
$$

Note on proof. The first step is to show that almost surely $\mu$ is exact dimensional (that is $\lim _{r \rightarrow 0} \log \mu(B(x, r)) /-\log r$ exists and takes a constant value $\mu$-almost 
everywhere), and also that for almost all $\theta, \operatorname{proj}_{\theta} \mu$ is exact dimensional with $\operatorname{dim}_{H} \operatorname{proj}_{\theta} \mu=\min \left\{\operatorname{dim}_{H} \mu, 1\right\}$. This is a random extension of the deterministic result of Feng and $\mathrm{Hu}$ [11], and uses an ergodic-theoretic argument to show that a natural 'shift-like' operator $T$ on the set comprising sequences and random variables

$$
\Omega=\left\{\left(i_{i}, i_{2}, \ldots\right),\left(W^{i_{1}, \ldots, i_{k}}: 1 \leq i_{j} \leq m, 1 \leq k<\infty\right)\right\}
$$

is invariant and ergodic with respect to the natural 'Peyrière measure' on $\Omega$. The space $\Omega$ and operator $T$ may then be extended to incorporate a rotation element which is ergodic by the compact group extension theorem.

The rest of the argument roughly follows that in [15]. Fix $0<\rho<1$. For each $q \in \mathbb{N}$, the IFS

$$
\mathscr{I}_{\rho^{q}}=\left\{f_{i_{1}} f_{i_{2}} \cdots f_{i_{k}}: r_{i_{1}} r_{i_{2}} \cdots r_{i_{k-1}}>\rho^{q} \geq r_{i_{1}} r_{i_{2}} \cdots r_{i_{k}}\right\}
$$

formed by compositions of the original contractions defines the same attractor $E$. Moreover, redefining the random vectors $W$ appropriately for this new IFS $\mathscr{I}_{\rho^{q}}$ we can get the same distribution of cascade measures $\mu$ on $E$. With $H_{r}$ denoting the ' $r$-scale entropy' for the corresponding map $T_{\rho^{q}}$, almost surely

$$
\begin{aligned}
\operatorname{dim}_{H} \operatorname{proj}_{\theta} \mu & \geq \frac{\mathbb{E}\left(H_{\rho q}\left(\operatorname{proj}_{\theta} \mu\right)\right)}{q \log (1 / \rho)-c}-O(1 / q) \quad \text { for all } \theta \\
& \rightarrow \operatorname{dim}_{H} \operatorname{proj}_{\theta} \mu \quad \text { as } q \rightarrow \infty \\
& =\min \left\{\operatorname{dim}_{H} \mu, 1\right\} \quad \text { for almost all } \theta .
\end{aligned}
$$

The proof is completed by using the lower-semicontinuity of $H_{\rho^{q}}$ together with a version of Marstrand's projection theorem for the dimension of projections of a measure in almost all directions.

The projection result for percolation on self-similar sets is an easy corollary.

Proof of Theorem 3.2 Let $s$ be such that $\sum_{i=1}^{m} r_{i}^{s} p_{i}=1$, so that conditional on nonextinction, $\operatorname{dim}_{H} E_{\mathbf{p}}=s$ almost surely. In Theorem 3.3 take

$$
W=\left(W_{1}, \ldots, W_{m}\right)=\left(r_{1}^{s} X_{1}, \ldots, r_{m}^{s} X_{m}\right)
$$

where $X_{1}, \ldots, X_{m}$ are independent with

$$
X_{i}=\left\{\begin{array}{l}
1 \text { with probability } p_{i} \\
0 \text { with probability } 1-p_{i}
\end{array}\right.
$$

A straightforward check establishes that, almost surely conditional on non-extinction, $\operatorname{dim}_{H} \mu=s$, so by Theorem 3.3, for all $\theta$,

$$
\operatorname{dim}_{H} \operatorname{proj}_{\theta} E_{\mathbf{p}} \geq \operatorname{dim}_{H} \operatorname{proj}_{\theta} \mu=\min \left\{\operatorname{dim}_{H} \mu, 1\right\}=\min \left\{\operatorname{dim}_{H} E_{\mathbf{p}}, 1\right\} .
$$

As with deterministic self-similar sets of dimension greater than 1, for percolation on self-similar sets we cannot quite guarantee projections of positive length in 

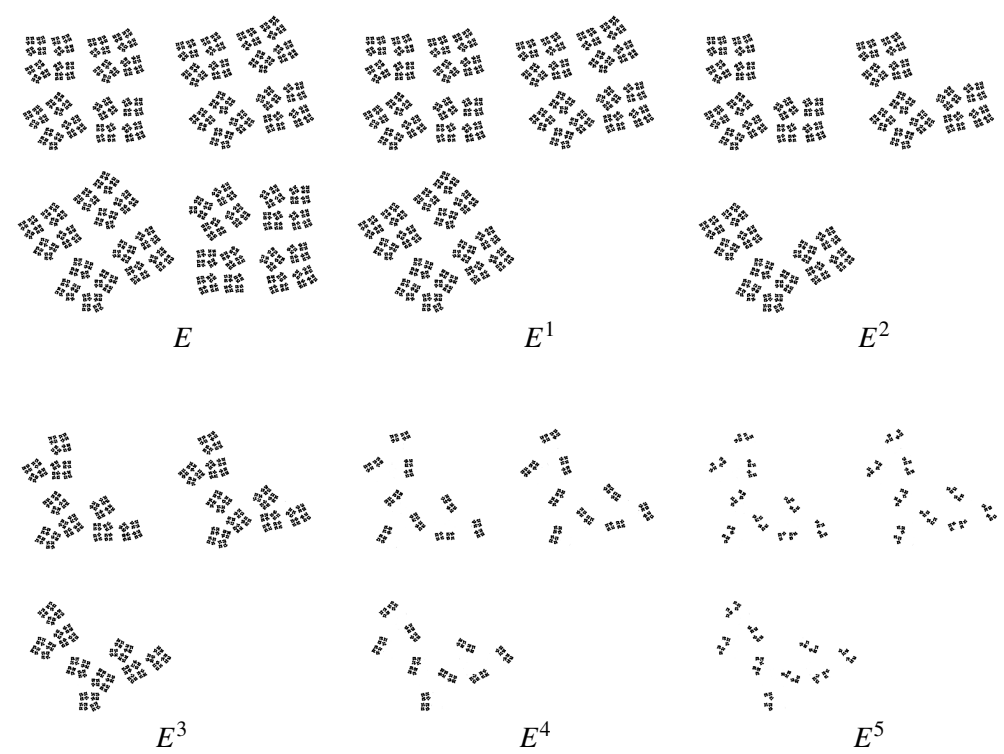

Fig. 5 Homogeneous percolation on a self-similar fractal $E$

all directions. However, the following analogue of Theorem 2.3 holds for subsets of $E$ obtained by a variant on the percolation process. We call a process to select a hierarchy of subsets of $E$ homogeneous percolation if at each stage of the construction, the same (random) pattern of subsets of each remaining component is selected, that is if for each component $\left\{f_{i_{1}} \cdots f_{i_{k}}(E)\right\}$ remaining at the $k$ th level of the process, the subsets of $\left\{f_{i_{1}} \cdots f_{i_{k}} f_{1}(E), \ldots, f_{i_{1}} \cdots f_{i_{k}} f_{m}(E)\right\}$ selected at the $(k+1)$ th stage depend only on $k$ (see Fig. 5).

Theorem 3.4. [8] Let $E$ be a self-similar set with dense rotations satisfying OSC and let $E_{\mathbf{p}} \subset E$ be obtained by homogeneous percolation defined by an appropriate random vector of probabilities such that, conditional on non-extinction, $\operatorname{dim}_{H} E_{\mathbf{p}}=s$ where $1<s<2$. Then there is a set of directions $\Theta \subset[0, \pi)$ of Hausdorff dimension 0 such that, almost surely, $\mathscr{L}\left(\operatorname{proj}_{\theta} E_{\mathbf{p}}\right)>0$ for all $\theta \notin \Theta$.

Note on proof. As before, this is proved by considering projections of suitable random measures. To fit in with homogeneous percolation, we take $W^{i_{1}, \ldots, i_{k}}=W^{(k)}$ for each $\left(i_{1}, \ldots, i_{k}\right)$ in (6) where $\left\{W^{(k)}\right\}_{k=1}^{\infty}$ are i.i.d. copies of a suitable vector of probabilities $W$, and thus define a random measure $\mu$ on the homogeneous percolation set $E_{\mathbf{p}}$. The proof, which develops that in [36], considers projections of $\mu$ onto lines by examining the decay of the Fourier transform $\widehat{\mu}$. The fact that the measures correspond to homogeneous percolation enables the Fourier transform to be expressed as a convolution of random measures. In particular it may be decomposed as $\widehat{\mu}(\xi)=\widehat{\mu_{0}}(\xi) \widehat{\mu_{1}}(\xi)\left(\xi \in \mathbb{R}^{2}\right)$ in such a way that, almost surely, $\operatorname{dim}_{H} \operatorname{proj}_{\theta} \mu_{0}=1$ for all $\theta$, and for all $\theta$ except for a set of dimension $0,\left|\widehat{\operatorname{proj}_{\theta} \mu_{1}}(\xi)\right| \leq c|\xi|^{-\varepsilon}$ for 
some $\varepsilon>0$, which together imply that $\operatorname{proj}_{\theta} \mu$ is absolutely continuous and that its support has positive Lebesgue measure.

\section{Using percolation to analyse sections of deterministic sets}

In this section we show how to derive dimensional properties of sections of a $d e$ terministic self-similar set $E$ using the conclusions of Section 3 on projections of random percolation subsets of $E$.

To find the Hausdorff dimension (or Hausdorff measure) of subsets of a selfsimilar set $E$ it is enough to take covers by the basic sets of the iterative construction of $E$, that is sets of the form $U_{\mathbf{i}}=f_{i_{1}} \circ \cdots \circ f_{i_{k}}(E)$ where $\mathbf{i}=i_{1}, \ldots, i_{k}$. In particular, for $F \subset E$,

$$
\begin{array}{r}
\operatorname{dim}_{H} F=\inf \left\{s: \text { for all } \varepsilon>0 \text { there are basic sets }\left\{U_{\mathbf{i}}\right\}_{\mathbf{i} \in \mathscr{I}}\right. \\
\text { with } \left.F \subset \bigcup_{\mathbf{i} \in \mathscr{I}} U_{\mathbf{i}} \text { and } \sum_{\mathbf{i} \in \mathscr{I}}\left(\operatorname{diam} U_{\mathbf{i}}\right)^{s}<\varepsilon\right\} .
\end{array}
$$

The following lemma shows how percolation can be used to test the dimension of non-random subsets of self-similar sets. We consider the percolation process described in Section 3 on the self-similar set $E$, using the vector of probabilities $\mathbf{p}=\left(p_{1}, \ldots, p_{m}\right)$. Note that the probability that a basic set $U_{\mathbf{i}}$ survives the percolation process is $p_{i_{1}} \cdots p_{i_{k}}$ where $\mathbf{i}=\left(i_{1}, \ldots i_{k}\right)$.

Lemma 4.1. Let $E$ be a self-similar set with basic sets $\left\{U_{\mathbf{i}}\right\}$. Let $E_{\mathbf{p}} \subset E$ be the random set obtained by percolation with vector of probabilities $\mathbf{p}$, and suppose that for some $\alpha>0$,

$$
\mathbb{P}\left\{U_{\mathbf{i}} \text { survives the percolation process }\right\} \leq c\left(\operatorname{diam} U_{\mathbf{i}}\right)^{\alpha} \text { for all } \mathbf{i} .
$$

If $F \subset E$ and $\operatorname{dim}_{H} F<\alpha$ then $E_{\mathbf{p}} \cap F=\emptyset$ almost surely.

Proof. With $\operatorname{dim}_{H} F<\alpha$, for all $\varepsilon>0$ we may find a set of words $\mathscr{I}$ such that

$$
F \subset \bigcup_{\mathbf{i} \in \mathscr{I}} U_{\mathbf{i}} \quad \text { and } \quad \sum_{\mathbf{i} \in \mathscr{I}}\left(\operatorname{diam} U_{\mathbf{i}}\right)^{\alpha}<\varepsilon .
$$

Then

$$
\begin{aligned}
\mathbb{E}\left(\#\left\{\mathbf{i} \in \mathscr{I}: E_{\mathbf{p}} \cap U_{\mathbf{i}} \neq \emptyset\right\}\right) & \leq \sum_{\mathbf{i} \in \mathscr{I}} \mathbb{P}\left\{U_{\mathbf{i}} \text { survives the percolation process }\right\} \\
& \leq c \sum_{\mathbf{i} \in \mathscr{I}}\left(\operatorname{diam} U_{\mathbf{i}}\right)^{\alpha}<c \varepsilon
\end{aligned}
$$

so

$$
\begin{aligned}
\mathbb{P}\left(E_{\mathbf{p}} \cap F \neq \emptyset\right) \leq \mathbb{P}\left(E_{\mathbf{p}} \cap \bigcup_{\mathbf{i} \in \mathscr{I}} U_{\mathbf{i}} \neq \emptyset\right) \\
\quad=\mathbb{P}\left(\#\left\{\mathbf{i} \in \mathscr{I}: E_{\mathbf{p}} \cap U_{\mathbf{i}} \neq \emptyset\right\} \geq 1\right)<c \varepsilon .
\end{aligned}
$$


This is true for all $\varepsilon>0$, so $\mathbb{P}\left(E_{\mathbf{p}} \cap F \neq \emptyset\right)=0$.

We apply the above lemma taking $F$ to be the sections $E \cap \operatorname{proj}_{\theta}^{-1} a$ of $E$.

Lemma 4.2. Let $E$ be a self-similar set with basic sets $\left\{U_{\mathbf{i}}\right\}$. Let $E_{\mathbf{p}} \subset E$ be the random set obtained by percolation with vector of probabilities $\mathbf{p}$, and suppose that for some $\alpha>0$,

$\mathbb{P}\left\{U_{\mathbf{i}}\right.$ survives the percolation process $\} \leq c\left(\operatorname{diam} U_{\mathbf{i}}\right)^{\alpha}$ for all $\mathbf{i}$.

For each $\theta$, if

$$
\mathbb{P}\left\{\mathscr{L}\left(\operatorname{proj}_{\theta} E_{\mathbf{p}}\right)>0\right\}>0,
$$

then

$$
\mathscr{L}\left\{a \in L_{\theta}: \operatorname{dim}_{H}\left(E \cap \operatorname{proj}_{\theta}^{-1} a\right) \geq \alpha\right\}>0 .
$$

Proof. Let

$$
S_{\theta}=\left\{a \in L_{\theta}: \operatorname{dim}_{H}\left(E \cap \operatorname{proj}_{\theta}^{-1} a\right)<\alpha\right\} .
$$

For each $a \in S_{\theta}$, taking $F=E \cap \operatorname{proj}_{\theta}^{-1} a$ in the previous lemma,

$$
E_{\mathbf{p}} \cap \operatorname{proj}_{\theta}^{-1} a=E_{\mathbf{p}} \cap E \cap \operatorname{proj}_{\theta}^{-1} a=\emptyset
$$

almost surely. In other words, for each $a \in S_{\theta}$ we have that $a \notin \operatorname{proj}_{\theta} E_{\mathbf{p}}$ with probability 1 . By Fubini's theorem, with probability $1, a \notin \operatorname{proj}_{\theta} E_{\mathbf{p}}$ for $\mathscr{L}$-almost all $a \in S_{\theta}$. Hence, with positive probability,

$$
0<\mathscr{L}\left(\operatorname{proj}_{\theta} E_{\mathbf{p}}\right)=\mathscr{L}\left(\left(\operatorname{proj}_{\theta} E_{\mathbf{p}}\right) \backslash S_{\theta}\right) \leq \mathscr{L}\left(\left(\operatorname{proj}_{\theta} E\right) \backslash S_{\theta}\right),
$$

from which the conclusion follows.

Lemma 4.2 allows us to deduce weak dimension conservation from Theorem 3.4 on projections of percolation sets.

Theorem 4.3. [8] Let $E \subset \mathbb{R}^{2}$ be a self-similar set with dense rotations satisfying OSC with $1<\operatorname{dim}_{H} E \leq 2$. Then for all $\varepsilon>0$,

$$
\mathscr{L}\left\{a \in L_{\theta}: \operatorname{dim}_{H}\left(E \cap \operatorname{proj}_{\theta}^{-1} a\right) \geq \operatorname{dim}_{H} E-1-\varepsilon\right\}>0
$$

for all $\theta$ except for a set of $\theta$ of Hausdorff dimension 0.

Proof. Let $\operatorname{dim}_{H} E=s>1$ and let $0<\varepsilon<s-1$. Define percolation on $E$ as above using the probabilities $p_{i}=r_{i}^{s-1-\varepsilon}(1 \leq i \leq m)$. Then with positive probability $E_{\mathbf{p}} \neq$ $\emptyset$, conditional on which $\operatorname{dim}_{H} E_{\mathbf{p}}=t$ where $1=\sum_{i=1}^{m} p_{i} r_{i}^{t}=\sum_{i=1}^{m} r_{i}^{s-1-\varepsilon} r_{i}^{t}$; since $\sum_{i=1}^{m} r_{i}^{s}=1$ this implies $\operatorname{dim}_{H} E_{\mathbf{p}}=t=1+\varepsilon$. Then

$\mathbb{P}\left\{U_{\mathbf{i}}\right.$ survives the percolation process $\}$

$$
=p_{i_{1}} \cdots p_{i_{k}}=\left(r_{i_{1}} \cdots r_{i_{k}}\right)^{s-1-\varepsilon}=c\left(\operatorname{diam} U_{\mathbf{i}}\right)^{s-1-\varepsilon} .
$$


By Theorem 3.4 there is a set of directions $\Theta$ with $\operatorname{dim}_{H} \Theta=0$ such that, with positive probability $\mathscr{L}\left(\operatorname{proj}_{\theta} E_{\mathbf{p}}\right)>0$ for all $\theta \notin \Theta$. For such $\theta$, taking $\alpha=s-1-\varepsilon$ in Lemma 4.2, gives (7).

It would be nice to eliminate the exceptional set of $\theta$ in the above theorem but this would also require doing so in Theorem 2.3. We can obtain a result on dimension of sections for all $\theta$ using Theorem 3.2. However, this only guarantees that the projections of a percolating set with $\operatorname{dim}_{H} E_{\mathbf{p}}>1$ have dimension 1 rather than positive Lebesgue measure, so that the set of sections in each direction which are 'large' is more limited. A further consequence of not being able to work using the Lebesgue measure of projections is that we can only get bounds for the lower box-counting dimension $\underline{\operatorname{dim}}_{B}$ of sections, and also an additional condition on the projections is needed.

Theorem 4.4. [8] Let $E \subset \mathbb{R}^{2}$ be a self-similar set with dense rotations satisfying OSC with $1<\operatorname{dim}_{H} E \leq 2$. Suppose either $E$ is connected or $\operatorname{proj}_{\theta} E$ is an interval for all $\theta$. Then for all $\varepsilon>0$

$$
\operatorname{dim}_{H}\left\{a \in L_{\theta}: \underline{\operatorname{dim}}_{B}\left(E \cap \operatorname{proj}_{\theta}^{-1} a\right) \geq \operatorname{dim}_{H} E-1-\varepsilon\right\}=1
$$

for all $\theta \in[0, \pi)$.

Note on proof. The proof very roughly follows the lines of that of Theorem 4.3, but taking Theorem 3.2 as the starting point. The extra restrictions here are because there is no convenient $\sigma$-finite measure available to play the role taken by Lebesgue measure in Lemma 4.2 which enabled Fubini's theorem to be applied.

The method can also be used to deduce weak dimension conservation for Mandelbrot percolation, described at the start of Section 3, from its known projection properties.

Theorem 4.5. [8] Let $E_{p}$ be the Mandelbrot percolation set obtained by dividing squares into $M \times M$ subsquares, each square being retained with probability $p>$ $1 / M^{2}$. Then, for all $\varepsilon>0$, conditional on $E_{p} \neq \emptyset$,

$$
\mathscr{L}\left\{a \in L_{\theta}: \operatorname{dim}_{H}\left(E_{p} \cap \operatorname{proj}_{\theta}^{-1} a\right) \geq \operatorname{dim}_{H} E_{p}-1-\varepsilon\right\}>0
$$

for all $\theta$.

Note on proof. The proof has a similar structure to the previous two theorems, starting with the projection dimensions of Mandelbrot percolation stated in Theorem 3.1. We then perform percolation with probability $q$ on $E_{p}$ and use that the intersection of two independent percolation sets $E_{p} \cap E_{q}$ has the same distribution as the single percolation set $E_{p q}$ constructed by retaining each square with probability $p q$.

Finally we remark that Shmerkin and Suomala [37] have recently introduced a very general theory showing that for a class of random measures, termed spatially independent martingales, strong results hold for dimensions of projections and sections of the measures, and thus of underlying sets, with the conclusions holding 
almost surely for projections in all directions. Such spatially independent measures include measures based on Mandelbrot percolation on squares, and in particular their approach can show that (8) is valid with $\varepsilon=0$.

\section{References}

1. J. Barral and B. Mandelbrot. Random multiplicative multifractal measures. II: Nondegeneracy, moments, dimension, and multifractal analysis for random multiplicative measures. In A Jubilee of Benoit Mandelbrot, Proc. Symp. in Pure Math., 72, pp. 17-52. AMS, Providence, RI, 2004.

2. B. Bárány, A. Ferguson and K. Simon. Slicing the Sierpiński gasket. Nonlinearity, 25: 17531770, 2012.

3. M. Dekking. Random Cantor sets and their projections. In Fractal Geometry and Stochastics IV, Progr. Probab., 61, pp. 269-284. Birkhäuser Verlag, Basel, 2009.

4. P. Erdős. On a family of symmetric Bernoulli convolutions, Amer. J. Math., 61: 974-976, 1935.

5. K. J. Falconer. Hausdorff dimension and the exceptional set of projections. Math. Proc. Cambridge Philos. Soc., 100: 559-582, 1986.

6. K. J. Falconer. Fractal Geometry - Mathematical Foundations and Applications. John Wiley \& Sons Ltd., Chichester, 3rd Ed., 2014.

7. K. J. Falconer and X. Jin. Exact dimensionality and projections of random self-similar measures and sets. J. Lond. Math. Soc. (2), 90, 388-412, 2014.

8. K. J. Falconer and X. Jin. Dimension conservation for self-similar sets and fractal percolation. Int. Math. Res. Notes, 24,13260-13289, 2015.

9. A. Farkas. Projections of self-similar sets with no separation condition, Israel J. Math., to appear, 2016, arXiv:1307.2841.

10. H. Furstenberg. Ergodic fractal measures and dimension conservation. Ergodic Theory Dynam. Systems, 28: 405-422, 2008.

11. D.-J. Feng and H. Hu. Dimension theory of iterated function systems. Comm. Pure Appl. Math., 62: 1435-1500, 2009.

12. H. Furstenberg. Ergodic Theory and Fractal Geometry, American Mathematical Society \& Conference Board of Mathematical Sciences, 2014.

13. M. Hochman. Dynamics on fractals and fractal distributions, arXiv:1008.3731v2, 2013.

14. M. Hochman. On self-similar sets with overlaps and inverse theorems for entropy, Ann. of Math.(2), 180: 773-822, 2014.

15. M. Hochman and P. Shmerkin. Local entropy averages and projections of fractal measures. Ann. of Math.(2), 175: 1001-1059, 2012.

16. J. Hutchinson. Fractals and self-similarity. Indiana Univ. Math. J., 30: 713-747, 1981.

17. J.-P. Kahane. Sur la distribution de certaines séries aléatoires. In Colloque de Théorie des Nombres, Univ. Bordeaux, 1969), pp 119-122. Bull. Soc. Math. France, Mém. 25, Soc. Math. France, Paris, 1971.

18. J.-P. Kahane. Positive martingales and random measures. Chinese Ann. Math. Ser. B, 8: 1-12, 1987.

19. J.-P. Kahane and J. Peyrière. Sur certaines martingales de B. Mandelbrot. Adv. Math. 22: 131-145, 1976.

20. R. Kaufman. On Hausdorff dimension of projections. Mathematika, 15: 153-155, 1968.

21. R. Kenyon. Projecting the one-dimensional Sierpinski gasket, Israel J. Math. 97: 221-238, 1997.

22. Q.-H. Liu, L.-F. Xi. and Y.-F. Zhao. Dimensions of intersections of the Sierpinski carpet with lines of rational slopes. Proc. Edinb. Math. Soc. (2), 50: 411-27, 2007. 
23. B. Mandelbrot. Intermittent turbulence in self-similar cascades: divergence of high moments and dimension of the carrier. J. Fluid Mech., 62: 331-333, 1974.

24. A. Manning and K. Simon. Dimension of slices through the Sierpinski carpet. Trans. Amer. Math. Soc., 365: 213-250, 2013.

25. J. M. Marstrand. Some fundamental geometrical properties of plane sets of fractional dimensions. Proc. London Math. Soc.(3), 4: 257-302, 1954.

26. J. M. Marstrand. The dimension of Cartesian product sets. Proc. Cambridge Philos. Soc., 50:198-202, 1954.

27. P. Mattila. Hausdorff dimension, orthogonal projections and intersections with planes. Ann. Acad. Sci. Fenn. Ser. A I Math., 1: 227-244, 1975.

28. P. Mattila. Geometry of sets and measures in Euclidean spaces, Fractals and rectifiability. Cambridge Studies in Advanced Mathematics 44. Cambridge University Press, Cambridge, 1995.

29. T. Orponen. On the distance sets of self-similar sets. Nonlinearity, 6: 1919-1929, 2012.

30. Y. Peres, W. Schlag and B. Solomyak. Sixty years of Bernoulli convolutions. In Fractal geometry and Stochastics, II, Greifswald/Koserow, 1998, 46, Progr. Probab., pp 39-65. Birkhäuser, Basel, 2000.

31. Y. Peres and P. Shmerkin. Resonance between Cantor sets. Ergodic Theory Dynam. Systems., 29: 201-221, 2009.

32. J. Peyrière. Turbulence et dimension de Hausdorff. C. R. Acad. Sc. Paris. 278: 567-569, 1974.

33. M. Rams and K. Simon. The dimension of projections of fractal percolations. J. Stat. Phys., 154: 633-655, 2014.

34. M. Rams and K. Simon. The geometry of fractal percolation. In Geometry and Analysis of Fractals, pp 303-323. Springer, Heidelberg, 2014.

35. M. Rams and K. Simon. Projections of fractal percolations. Ergodic Theory Dynam. Systems., 35: 530-545, 2015.

36. P. Shmerkin and B. Solomyak. Absolute continuity of self-similar measures, their projections and concolutions. Trans. Amer. Math. Soc., 368, 5125-5151, 2016.

37. P. Shmerkin and V. Suomala. Spatially independent martingales, intersections, and applications. Mem. Amer. Math. Soc., to appear, 2016, arXiv:1409.6707.

38. K. Simon and L. Vágó Projections of Mandelbrot percolation in higher dimensions. In Fractals, Wavelets and their Applications, pp 175-190, Springer, Cham, 2014. 